\title{
Apparent resistivity and spectral induced polarization in the submarine environment
}

\author{
HERCULES DE SOUZA ${ }^{1}$ and EDSON E. S. SAMPAIO ${ }^{2}$ \\ ${ }^{1}$ Departamento de Magnetologia, Base Naval de Aratu, 40800-310 Salvador, Bahia, Brasil \\ ${ }^{2}$ Centro de Pesquisa em Geofísica e Geologia, Universidade Federal da Bahia \\ Campus Universitário de Ondina, Instituto de Geociências sala 312C, 40170-290 Salvador, Bahia, Brasil \\ Manuscript received on November 7, 2000; accepted for publication on May 15, 2001; \\ presented by Diogenes A. CAMPOS
}

\begin{abstract}
Relatively few investigations have employed electrical methods in the submarine environment, which may be promising for mineral deposits or threatened by environmental problems. We have measured the electric field using both disk and bar electrodes in the sea water at three different levels: sea surface, seven meters deep, and sea bottom at a depth of ten meters, employing a $2 \mathrm{~m}$ spacing dipole-dipole array with 7 array spacings of investigation, and 13 values of frequencies at steps of $\left(2^{\mathrm{N}}\right.$ hertz, $\left.\mathrm{N}=-2,-1,0,1,2, \ldots . .10\right)$. The measurement allowed the analysis of the electric field as a function of frequency and spacing, and of the spectral induced polarization. Modelling and interpretation of the apparent resistivity yielded a good fit with previous drilling data. Analysis of the spectrum of the complex apparent resistivity and the comparison with equivalent circuits, provided information about the grain size, the mineral composition and the major induced polarization phenomenon occurring below the sea. Therefore the result of the present research show the feasibility of measuring the variation of seawater resistivity in situ, as well as the resistivity of sea bottom sediments.
\end{abstract}

Key words: complex apparent resistivity, induced polarization, forward modeling, sea water.

\section{INTRODUCTION}

Electrical methods in applied geophysics can employ natural or artificial sources. Among the publications that have used artificial sources for the investigation of submarine environments during the last twenty years, we can mention (Edwards et al. 1981), (Chave and Cox 1982), (Edwards and Chave 1986), (Wynn 1988), and (Cheesman et al. 1987, 1990 and 1991). (Chave et al. 1991) designed new theoretical models for determining the electrical conductivity of rocks under the bottom of the sea, and (Baumgartner

Correspondence to: Edson E. S. Sampaio

E-mail: edson@cpgg.ufba.br
1996) obtained the electrical resistivity of sedimentary layers at the bottom of a lake.

The main problems for using electrical methods in the sea are the electromagnetic coupling and the attenuation because of the high conductivity of the seawater. Other sources of noise are spurious telluric currents, time variation of natural fields, and cultural noise, especially those caused by metallic objects near the receiving electrodes. The eddy currents that appear due to the electromagnetic field also delay the phase of the signals and mask the interpretation.

The objective of this research is to investigate the feasibility of establishing the electrical conduc- 
tivity and induced polarization effects of both the seawater and the bottom sediments in a shallow sea environment. The results of such a study are important for coastal engineering and environmental protection projects. We did the survey employing the dipole-dipole array and a multi-spectral system. In our analysis we fully considered the propagation of electromagnetic waves in conductive media and analyzed the electric field with pseudo-sections of the induced polarization spectrum using both the modulus and the phase of the complex apparent resistivity. Additionally we also computed the percent frequency effect (PFE) between $1 \mathrm{~Hz}$ and $8 \mathrm{~Hz}$. We also did forward and inverse modeling of the apparent resistivity data employing results previously obtained by (Dias 2000) and (Sato 2000).

\section{ACQUISITION AND ANALYSIS OF THE EXPERIMENTAL DATA}

We selected a place near the Naval Base of Aratu in Salvador, Bahia, Brazil for our experimental work. The place has the entire necessary infrastructure for the proposed search. Furthermore it is located in a small bay free of seawater turbulence, where the water layer is about $10 \mathrm{~m}$ deep. Laboratory measurements of the seawater conductivity at room temperature and direct-current gave the following average values: $4.90 \mathrm{~S} / \mathrm{m}$ at the sea surface; $5.06 \mathrm{~S} / \mathrm{m}$ at 7 $\mathrm{m}$ of depth; and $4.92 \mathrm{~S} / \mathrm{m}$ at the sea bottom. Besides frequency and conductivity, the propagation of an electromagnetic wave along the seawater depends on the magnetic permeability, $\mu$. $\simeq \mu_{0}=$ $4 \pi \times 10^{-7}$ henry $/ \mathrm{m}$, where $\mu_{0}$ is the magnetic permeability of vacuum, and the relative dielectric permittivity, $\epsilon$, which varies between 78 and 81 . Therefore for the seawater the skin depth in meters equals approximately to $225 / \sqrt{\mathrm{f}}$, $\mathrm{f}$ being the frequency in hertz. A drilling at the site gave the following data for the layers below the bottom of the sea: a $6 \mathrm{~m}$ thick unconsolidated sandy clay top layer; a second unconsolidated layer of silt with a thickness of 1 meter; and a thick third layer composed of sandstone and silt.

We positioned the lines of electrodes at three different levels: sea surface, middle of the water layer at $7 \mathrm{~m}$ below sea level, and sea bottom at 10 $m$ of depth. In order to fix the electrodes at each level, navy divers stretched and tied a non-metallic rope with $0.03 \mathrm{~m}$ of diameter to two pillars of the pier spaced of $50 \mathrm{~m}$. Subsequently the electrodes were tied to that rope. Figure 1 sketches the geological layers and the three levels of the lines of the electrodes. We constructed and used four types of electrodes to investigate the influence of the shape and of the dimension of the electrodes on the measurements. We employed steel electrodes with the following dimensions and forms: disk with a radius of $0.075 \mathrm{~m}$ and a thickness of $1.5 \times 10^{-3} \mathrm{~m}$; disk with a radius of $0.025 \mathrm{~m}$ and a thickness of $1.5 \times 10^{-3} \mathrm{~m}$; vertical bar with a length of $0.10 \mathrm{~m}$ and a diameter of $5.0 \times 10^{-3} \mathrm{~m}$; and vertical bar with a length of 0.03 $\mathrm{m}$ and a diameter of $5.0 \times 10^{-3} \mathrm{~m}$. Because all the electrodes gave similar results within the precision of the measurements, it will suffice to present the behavior of only one type of electrode per analysis.

The contact between the seawater electrolyte and the electrode metal may create potentials that vary with time. This constitutes an additional source of noise. In order to decrease this source of noise, (Wynn 1988) towed a non-polarizable pair of Ag$\mathrm{AgCl}$ receiving electrodes. According to (Petiau and Dupis 1980) steel electrodes are simpler and stronger. They are also much cheaper. They present a noise of $10 \mu \mathrm{V}$ for a stability time between half and one hour after its installation, and $4 \mu \mathrm{V}$ after 24 hour of installation, for frequencies below $1 \mathrm{~Hz}$. For frequencies above $1 \mathrm{~Hz}$ the electrode noise is lower than $2 \mu \mathrm{V}$ after 24 hours of installation. We monitored this noise and we always did our measurements 24 hours after placing the electrodes in the seawater, to take advantage of this stabilization time. To connect each electrode to the geophysical equipment, we used individual cables with grounded shield suitable for submarine application. They have one metallic shield on one semi-conductor layer. These layers serve to confine the electric field into the cable to minimize the coupling between the nearby cables and to eliminate the danger of electric shocks. We 


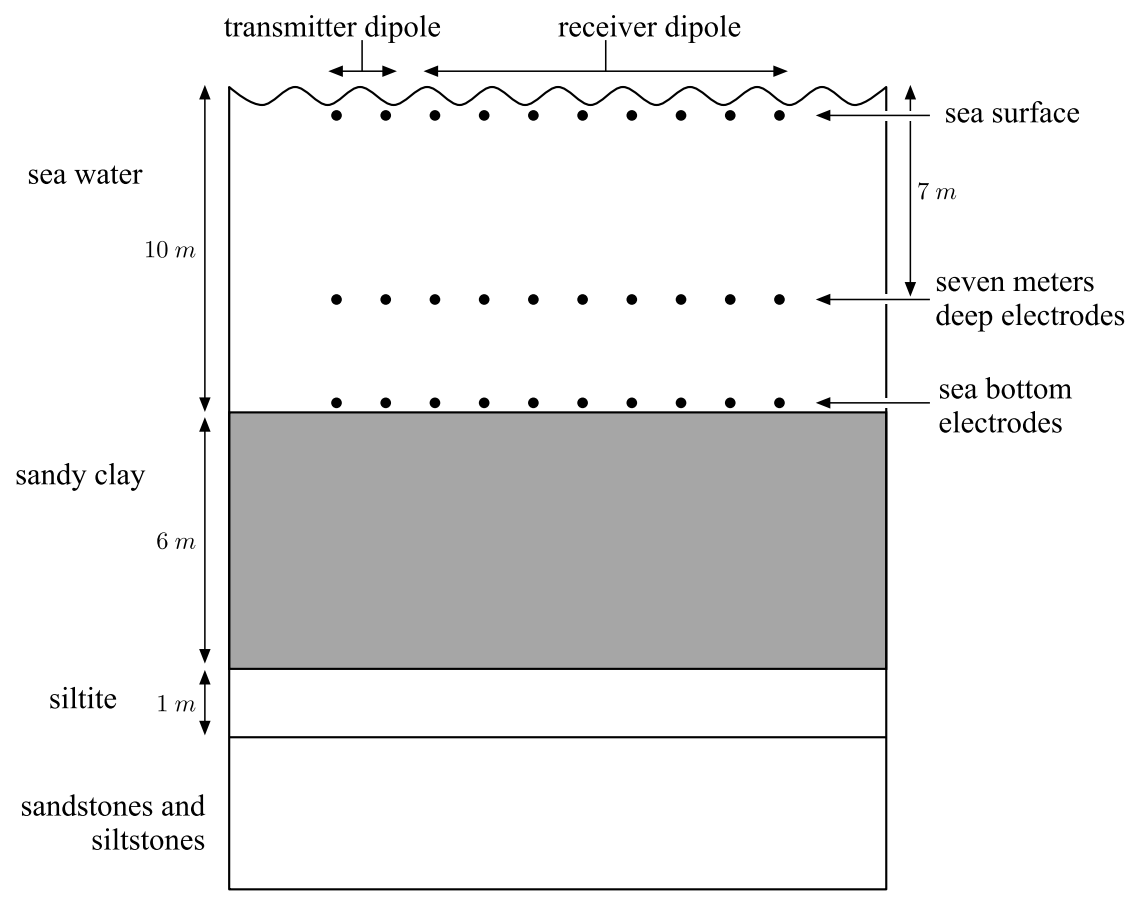

Fig. 1 - Sketch of the geological layers and of the three levels of the survey.

employed a TV-4 multi-spectral receiver of the SIP system manufactured by Phoenix Geophysics Limited. We calibrated the system before starting the measurements.

We employed the dipole-dipole array with a dipole length of $2 \mathrm{~m}$, with 7 different array spacing ( $\mathrm{n}=1,2, \ldots, 7)$ of investigation, and thirteen values of frequency, that ranged from $2^{-2}(0.25) \mathrm{Hz}$ to $2^{10}(1024) \mathrm{Hz}$ in steps of

$$
\left(2^{\mathrm{N}}, \mathrm{N}=-2,-1,0,1,2, \ldots \ldots 10\right) .
$$

This frequency range and a constant dipole length of $2 \mathrm{~m}$ for both the transmitter electrodes and the receiver electrodes were used for monitoring the electromagnetic coupling, and therefore select the appropriate frequency range for analyzing the induced polarization phenomenon. After the data acquisition the procedure also aimed to study the electric field behavior as a function of electrode type and depth in the water layer. All the data were first en- tered in a notebook and later transferred to a UNIXworkstation system for processing.

\section{PSEUDO-SECTION OF THE ELECTRIC FIELD}

The experimental data provided the analysis of the real and imaginary parts of the electric field via pseudo-sections as a function of frequency and spacing. This enabled us to analyze the parameters of the induced polarization. These data are presented on Figures 2, 3, and 4, for the disk electrode with a radius of $0.075 \mathrm{~m}$.

In Figures 2a, 3a and $4 \mathrm{a}$ we can see the real parts of the electric field for the three levels. The behavior of the real part of this field is uniform and shows vertical and lateral variations. Starting from $100 \mathrm{~Hz}$ there is a tendency of attenuation of the electric field mainly for spacing higher than 8 meters. This attenuation happens mainly because of the skin depth effect, but it is enhanced by the scattering at the sea surface. 
a)

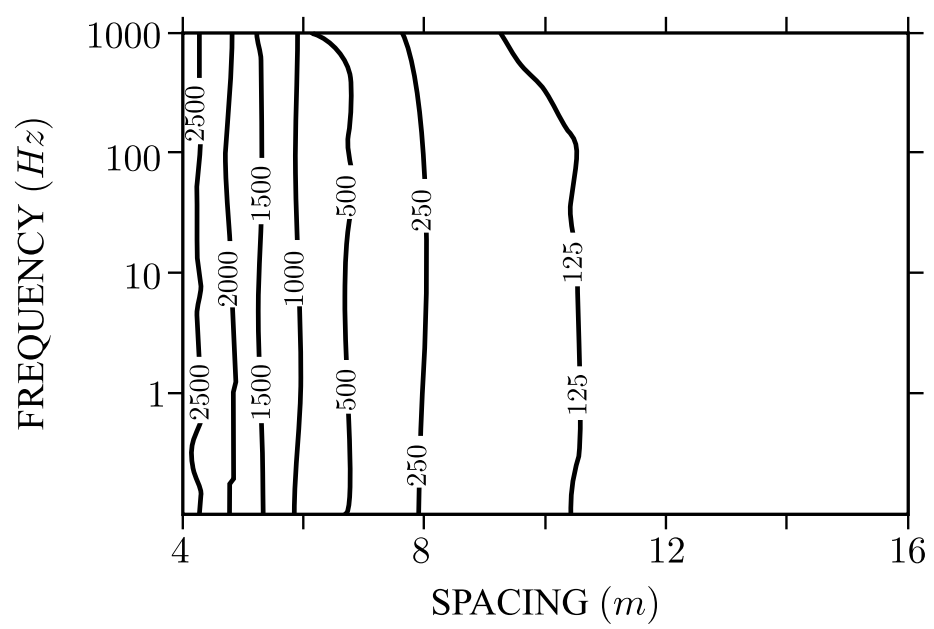

b)

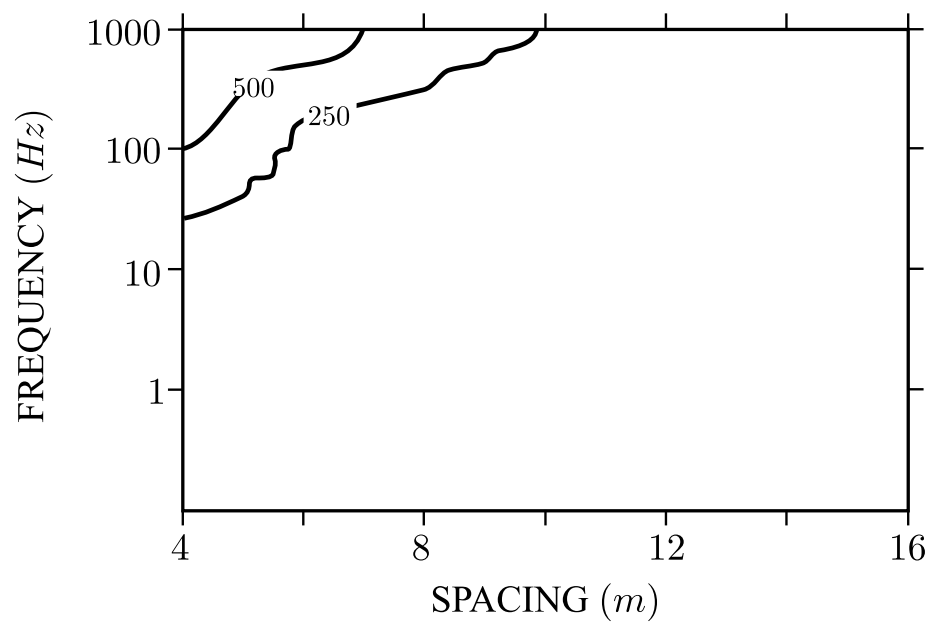

Fig. 2 - Variation of (a) the real part and (b) the imaginary part of the electric field on the sea surface, in $\mathrm{nV} / \mathrm{m} / \mathrm{mA}$ as a function of frequency and lateral spacing $(\mathrm{n}=1,2, \ldots, 7)$.

Figures $2 \mathrm{~b}, 3 \mathrm{~b}$ and $4 \mathrm{~b}$ show the imaginary part of the electric field. Its variation is mainly a function of frequency and not a function of spacing as it is for the real part. The imaginary part on the sea bottom is also higher than on the sea surface or in the middle of the water layer. This is also caused by the combination of the skin depth effect and the scattering at the sea surface.

\section{PARAMETERS OF SPECTRAL INDUCED POLARIZATION}

We computed the amplitude and the phase of the apparent resistivity function for the dipole-dipole array as a function of frequency for all the levels of investigation. The amplitude value corresponds to a half-space for the sea surface and to full-space for the other two levels. We represent these data for the vertical bar electrode with a length of $0.10 \mathrm{~m}$, not 
a)

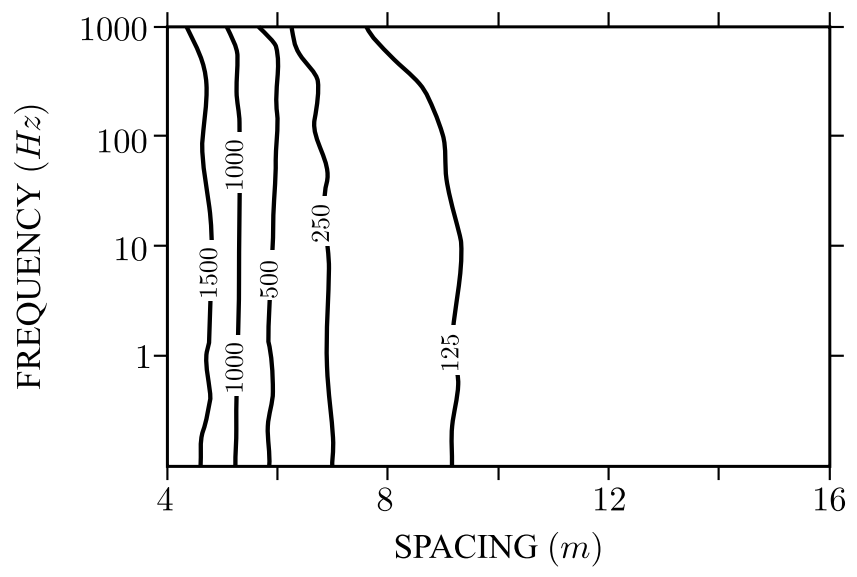

b)

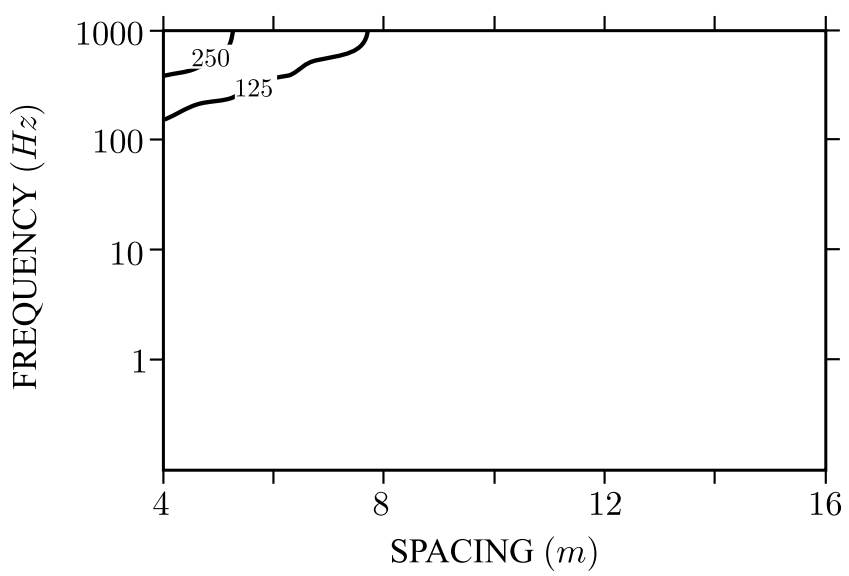

Fig. 3 - Variation of (a) the real part and (b) the imaginary part of the electric field at a depth of $7 \mathrm{~m}$, in $\mathrm{nV} / \mathrm{m} / \mathrm{mA}$, as a function of frequency and lateral spacing $(\mathrm{n}=1,2, \ldots, 7)$.

corrected for inductive coupling, on Figures 5, 6, and 7. Figures $5 \mathrm{a}, 6 \mathrm{a}$ and $7 \mathrm{a}$ show the behavior of the amplitude in $\Omega \cdot m$, and Figures $5 \mathrm{~b}, 6 \mathrm{~b}$, and $7 \mathrm{~b}$ show the behavior of the phase in mrad. In general the behavior in these figures is uniform below $64 \mathrm{~Hz}$.

Figure 5a shows the results for the electrodes placed on the sea surface. For array spacing 1 to 4 the curves show little fluctuations whereas for 5 to 7 and frequencies above $64 \mathrm{~Hz}$, the plot shows a noisy behavior. Probably the noise is related to power lines close to the site of measurement. On this same figure we obtained a maximum variation of $0.20 \Omega \cdot m$ and a minimum variation of $0.16 \Omega \cdot m$ for frequencies lower than $32 \mathrm{~Hz}$.

Figure 6a shows the results for the electrodes placed 7 meters below sea level. In this figure we obtained a maximum variation of $0.34 \Omega \cdot m$ and a minimum variation of $0.17 \Omega \cdot m$, below $64 \mathrm{~Hz}$. Figure 7 a shows the results for the electrodes at the sea bottom (10 meters deep). In this figure we obtained a maximum variation of the $0.34 \Omega \cdot m$ and a minimum variation of the $0.30 \Omega \cdot m$, also below $64 \mathrm{~Hz}$.

Except for one curve, the phase curves represented in Figures $5 b, 6 b$ and $7 b$ increase with 
a)

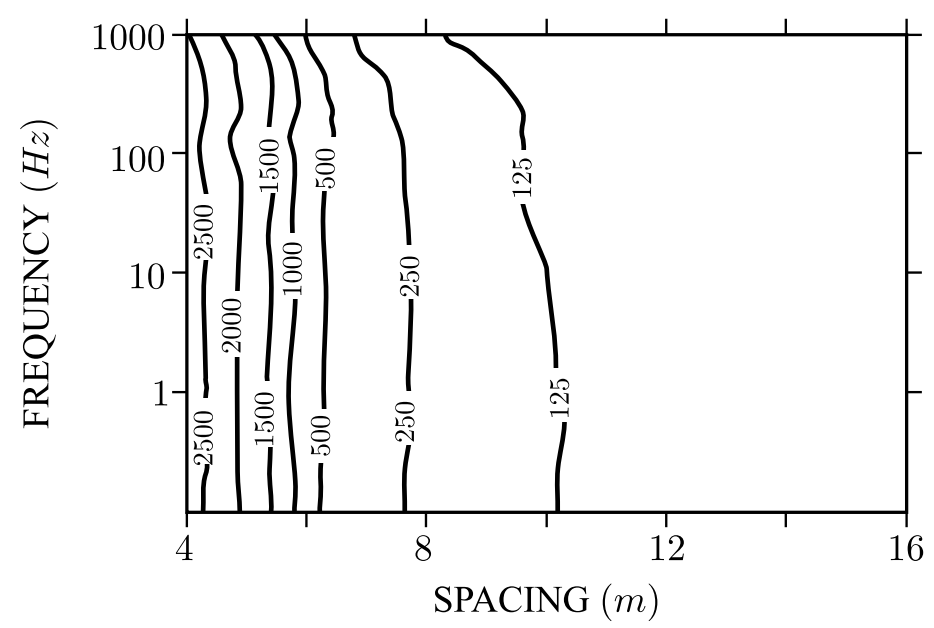

b)

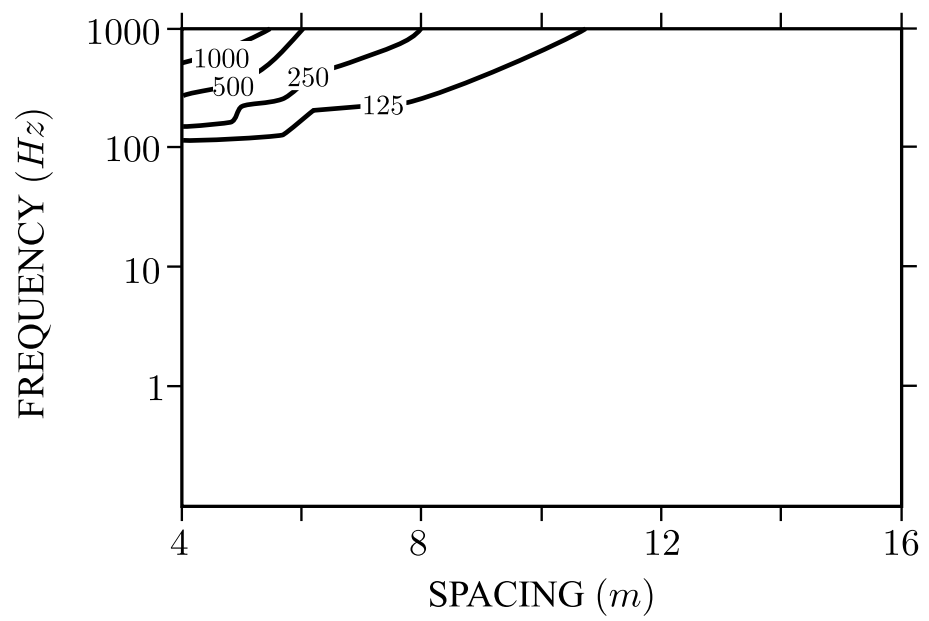

Fig. 4 - Variation of (a) the real part and (b) the imaginary part of the electric field at the sea bottom, in $\mathrm{nV} / \mathrm{m} / \mathrm{mA}$, as a function of frequency and lateral spacing $(\mathrm{n}=1,2, \ldots, 7)$.

frequency above $64 \mathrm{~Hz}$. This is typical of electromagnetic coupling (Ward 1990). The exception occurs only for the first array spacing of investigation with the electrodes on the surface of the sea. For this case we should have the largest coupling effect on the phase value due to the vicinity between the receiver electrodes and the transmitter electrodes. This anomalous behavior is caused probably because of the interaction with the location of the equipment.

In Figures 5-7 we observe a large variation of the apparent resistivity and phase curves for frequencies above $64 \mathrm{~Hz}$, which is more critical for the array spacing 5-7. This fact is due to the high conductivity of the medium and consequent dispersion of the electric current from the transmitter to the medium, resulting in a strong decrease of the signal with increasing frequency for the farther receiving electrodes. We can also see negative values of phase. (Nabighian and Elliot 1976) explain this behavior by an alteration produced by peculiar pattern of the geoelectric layers. 
a)

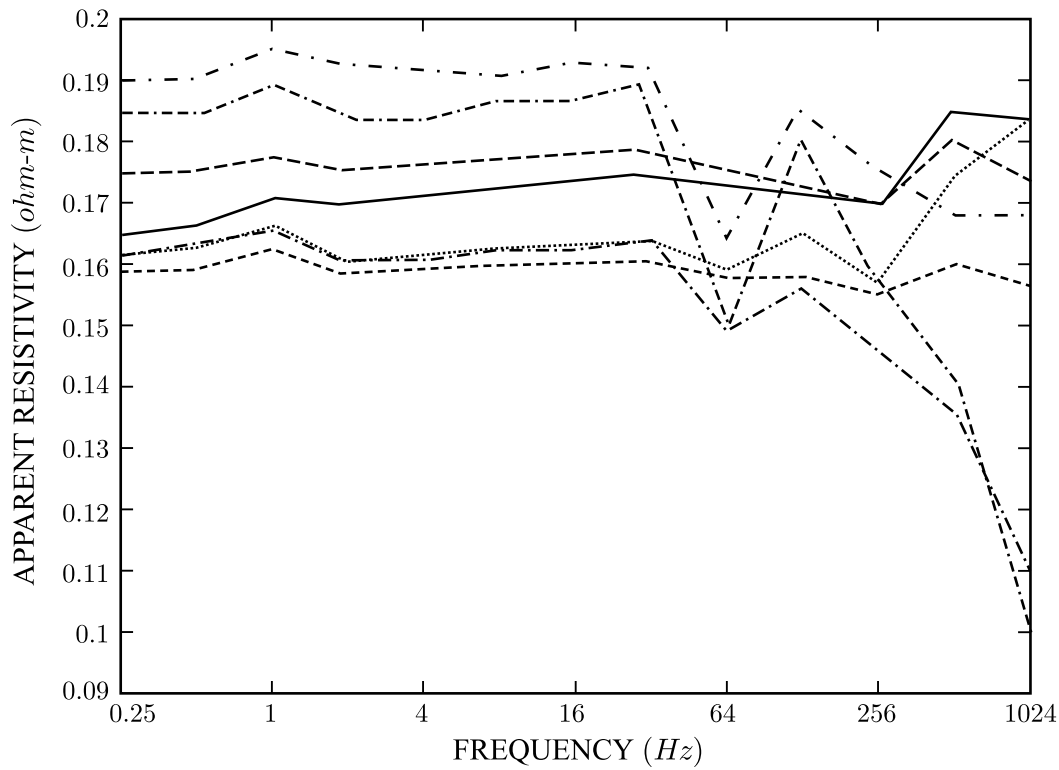

$n=$ array spacing

' $n=1$ '

' $n=2$ ' $-\mathbf{- - -}$

' $n=3$ ' $-\cdots-$

' $n=4$ '

' $n=5$ ' $-\cdot-\cdot-$

' $n=6$ ' - - - .

' $n=7$ ' - -....'

b)

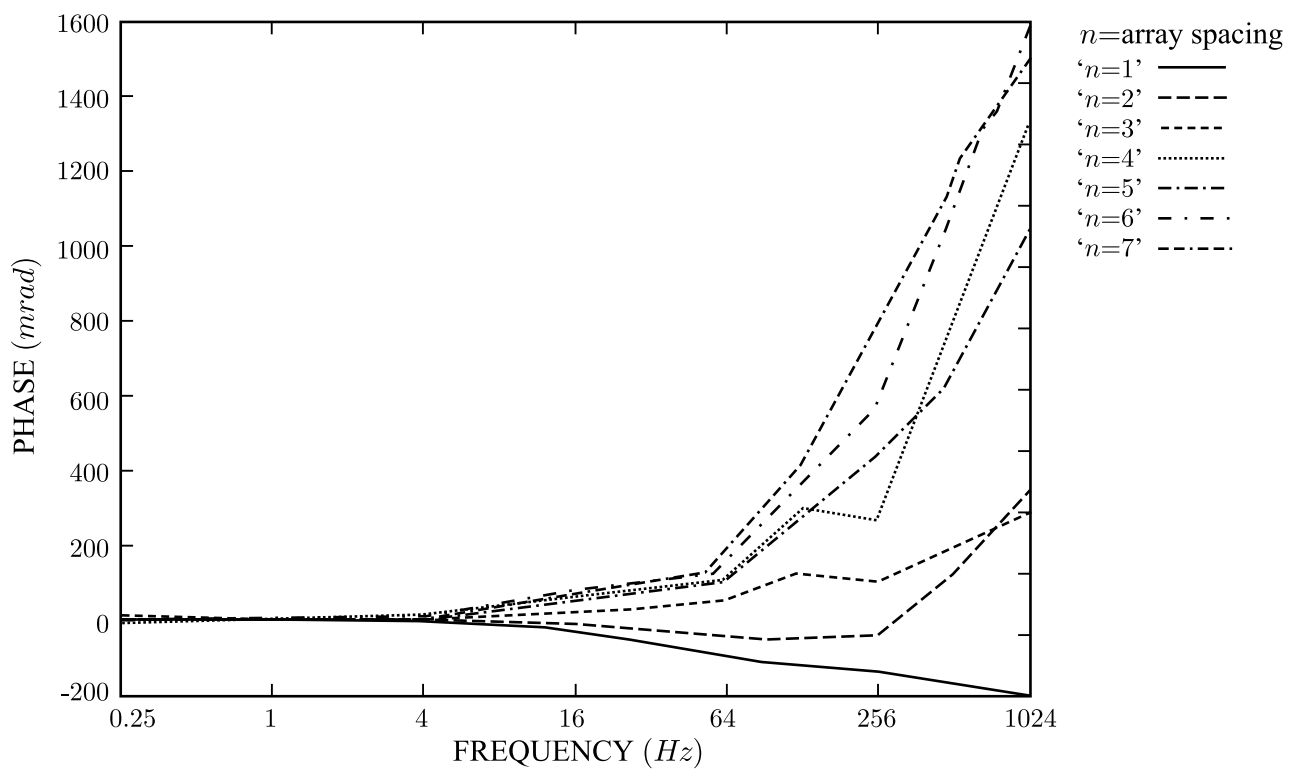

Fig. 5 - Behavior of (a) the amplitude and (b) the phase of the apparent resistivity on the sea surface, for the seven array spacing of investigation, and for the bar electrode $(0.10 \mathrm{~m})$.

(Hallof et al. 1979) state that the phase of the resistivity tends to show minimum values for a given frequency - the critical frequency. The value of this frequency is related to the grain size of the minerals showing polarization. If this critical frequency is not reached, as is the case shown in Figure $7 \mathrm{~b}$ for all levels of investigations, this is an indication of a smaller grain size of the minerals, possibly clays. 
a)

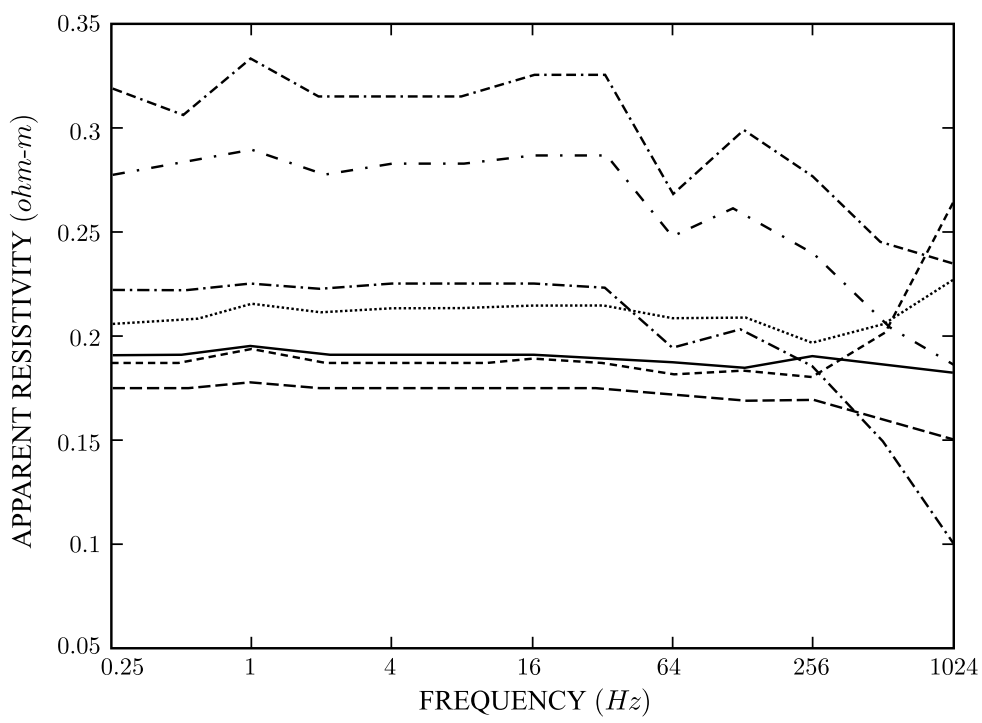

$n=$ array spacing

' $n=1$ '

' $n=2$ '

' $n=3$ ' $\cdots$

' $n=4$ ' $\quad \ldots . . . \cdots \cdots . . .$.

' $n=5$ ' $-\cdot \cdot-\cdot$

' $n=6$ ' - - -

' $n=7$ ' -...-.

b)

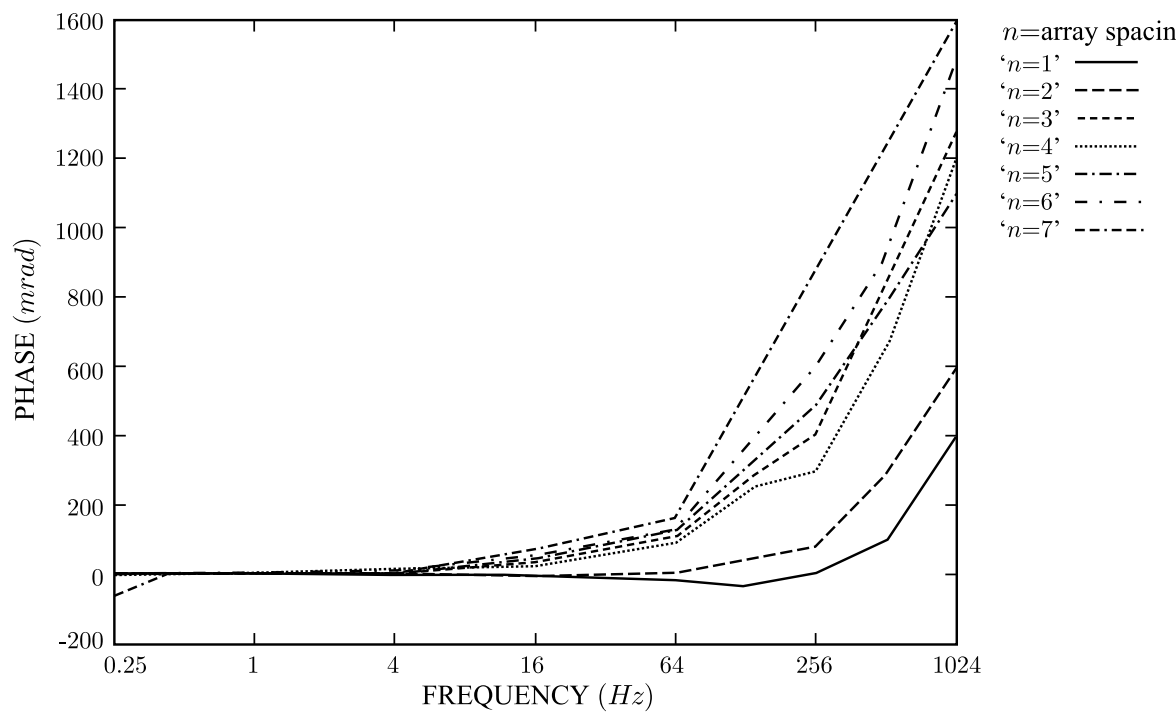

Fig. 6 - Behavior of (a) the amplitude and (b) the phase of the apparent resistivity $7 \mathrm{~m}$ deep, for the seven array spacing of investigation, and for the bar electrode $(0.10 \mathrm{~m})$.

\section{PERCENT FREQUENCY EFFECT}

We computed the Percent Frequency effect (PFE) employing the following expression:

$$
P F E=\frac{\rho_{1.0}-\rho_{8.0}}{\rho_{8.0}} \times 100 \%
$$

In equation (1) $\rho_{1.0}$ is the resistivity at $1.0 \mathrm{~Hz}$ and $\rho_{8.0}$ is the resistivity at $8.0 \mathrm{~Hz}$. They represent respectively the " $\mathrm{dc}$ " and the "ac" electrical resistivities. We have chosen these two frequency values to satisfy the following conditions: (i) that the value of the high ("ac") frequency is close to ten times the value of the low ("dc") frequency. (ii) That 
a)

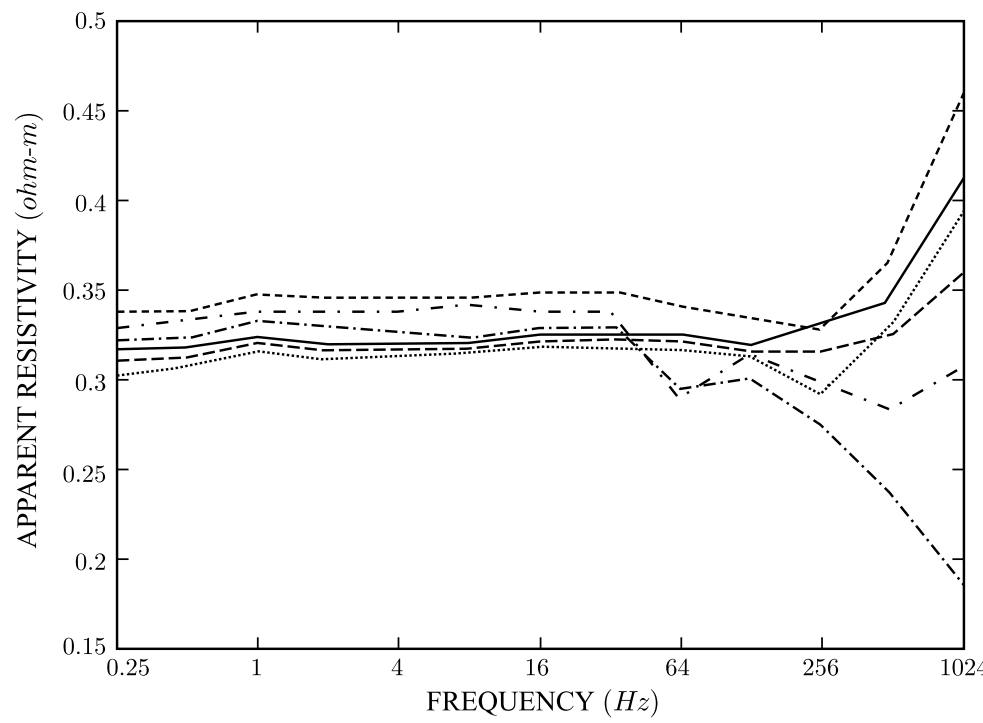

b)

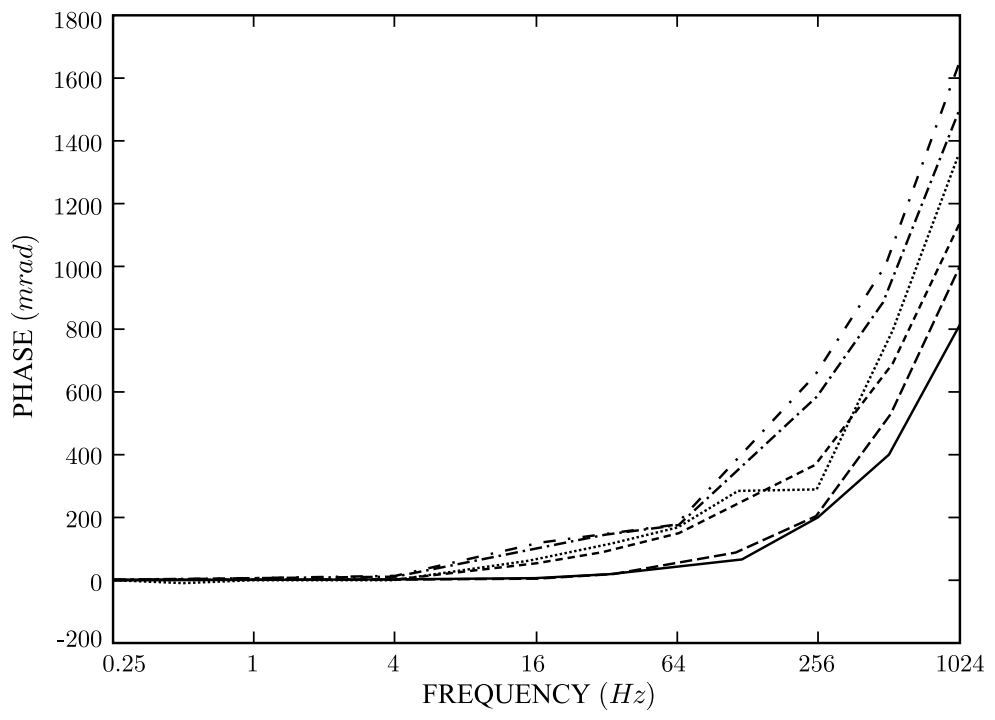

$n=$ array spacing

' $n=1$ '

' $n=2$ ' ----

' $n=3$ ' - ----

' $n=4$ '

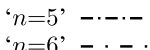

$n=$ array spacing

' $n=1$ '

' $n=2$ ' - - -

' $n=3$ '

' $n=4$ '

' $n=5$ '

' $n=6$ ' $-\cdot \cdot$.

Fig. 7 - Behavior of (a) the amplitude and (b) the phase of the apparent resistivity at the sea bottom, for the six array spacing of investigation, and for the bar electrode $(0.10 \mathrm{~m})$.

the "ac" frequency value be low enough not to be severely affected by the EM coupling, which causes abnormal increase of the phase value and decrease of the mutual impedance value. (iii) That the " $\mathrm{dc}$ ", frequency value be high enough not to be severely affected by the noise caused by flow, intermixing, and oscillation of the sea water at the Aratu Bay, with a period of about $2 \mathrm{~s}$. Probably this low frequency noise is the main cause for the maximum value of the apparent resistivity at $1.0 \mathrm{~Hz}$, and the negative phase values below $1 \mathrm{~Hz}$.

Table I shows the PFE values for the three seawater levels employing the vertical bar electrode with the length of $0.03 \mathrm{~m}$. Table I shows small 
positive values of PFE at the sea surface, seven meter deep, and the sea bottom. Except for $n=4$ and $n=5$, where the PFE on the sea surface and 7 meter depth reaches $1.3 \%, 1.4 \%$ and $2.2 \%$, the sea bottom yields the higher values of PFE, none of them above $2.5 \%$. According to (Lima and Sharma 1992) the frequencies of $1 \mathrm{~Hz}$ and $8 \mathrm{~Hz}$ are within the range of frequencies where the diffusion mechanism is the most important for polarization. Also previous drilling at the site yielded core samples of sediments containing disseminated clay. Therefore, this weak IP effect suggests the occurrence of membrane polarization due to the presence of clay in the sediments under the sea bottom.

\section{FORWARD MODELING}

We employed the algorithm developed by (Sato 2000) to compute forward models for the three sea levels of investigation. We used three layers for the initial model. The first layer has a resistivity of $0.19 \Omega \cdot m$ and a thickness of $10.5 \mathrm{~m}$, the second layer has a resistivity of $2.0 \Omega \cdot m$ and a thickness of 6.0 $\mathrm{m}$, and the third layer has a resistivity of $10.0 \Omega \cdot m$. The results of this modeling are shown on Figure 8 and indicate a good fitting between the experimental and the theoretical data. In order to minimize the effect of frequency we employed the data of the lowest frequency, $0.25 \mathrm{~Hz}$, to compare with the algorithm of (Sato 2000), which was developed for the directcurrent case. The data represented in Figure 8 have been obtained with a different type of electrode and a different value of frequency from the data represented in Table I, resulting in very different data for $\mathrm{n}=1$.

The values of the theoretical curve for the sea surface approach the experimental values with a slight tendency to grow for the larger spacing. The theoretical curve at $7 \mathrm{~m}$ depth fits better the experimental data. We observed a point of crossing between the theoretical curves for the sea surface and $7 \mathrm{~m}$ depth, both at an array spacing of 6 meters. The same crossing appears between the correspondent experimental curves at an array spacing of $7 \mathrm{~m}$. The theoretical curve of the sea bottom shows the same increase relative to the experimental data at a spacing of $6 \mathrm{~m}$, a small decrease for $10 \mathrm{~m}$ and tends to grow for distances larger than $10 \mathrm{~m}$.

The result obtained from this modeling emphasize the viability of the application of an electric method in the sea, because from the initial model it was possible to obtain a good fit between theoretical and the experimental curves.

\section{EQUIVALENT ANALOG CIRCUIT}

All rock samples are contaminated from the viewpoint of the physical chemist since the electrodes and electrolytes are anything but pure. Nevertheless, it is justifiable to employ equivalent circuits based on pure systems since a phenomenological explanation for rock behavior results (Ward 1990).

(Debye 1929) has formulated the phenomenon of polarization in rocks suitable for homogeneous substances. (Cole and Cole 1941) modified the expression of Debye on empirical grounds to describe inclusive composite substances. However their expression does not have a general validity. Subsequent authors have suggested models and analytic expression for the complex electric conductivity in rock based on electric analog circuits to describe the induced polarization phenomenon. Among them we mention (Dias 1972, 2000) and (Pelton et al. 1978). Dias' model, reproduced in Figure 9, is based on an analog fundamental circuit. It represents both the electrode and the membrane polarization phenomena, and it is based on the following equation:

where

$$
\begin{gathered}
\sigma=\sigma_{0}\left(1+\frac{\beta \alpha \lambda \sqrt{i \omega}}{1+\beta \lambda^{*} \sqrt{i \omega}}\right), \\
\lambda=1+\mu, \\
\lambda^{*}=1+(1-\delta) \mu, \\
\mu=i \omega \tau\left(1+\frac{\eta}{\sqrt{i \omega}}\right), \\
\alpha=m\left(\frac{1-\delta}{1-m}\right), \\
\beta=\frac{1}{\eta \delta} .
\end{gathered}
$$




\section{TABLE I}

Percent frequency effect (PFE) employing $1.0 \mathrm{~Hz}$ and 8.0 $\mathrm{Hz}$ and the vertical bar electrode $(\mathrm{L}=0.03 \mathrm{~m})$ for the given spreads $\mathbf{n}$ and positions.

\begin{tabular}{c|c|c|c|c|c|c}
\hline \multirow{2}{*}{$\mathrm{n}$} & \multicolumn{2}{|c|}{ Sea surface } & \multicolumn{2}{c|}{ Seven meters deep } & \multicolumn{2}{c}{ Sea bottom } \\
\cline { 2 - 7 } & ohm.m & PFE\% & ohm.m & PFE\% & ohm.m & PFE\% \\
\hline \multirow{2}{*}{1} & 0.142 & 0.0 & 0.206 & 1.0 & 0.300 & 1.7 \\
& 0.142 & & 0.204 & & 0.295 & \\
\hline 2 & 0.164 & 0.6 & 0.187 & 1.1 & 0.318 & 1.0 \\
& 0.163 & & 0.185 & & 0.315 & \\
\hline \multirow{2}{*}{3} & 0.153 & 0.7 & 0.199 & 1.0 & 0.332 & 1.2 \\
& 0.152 & & 0.197 & & 0.328 & \\
\hline 4 & 0.157 & 1.3 & 0.224 & 1.4 & 0.314 & 0.6 \\
& 0.155 & & 0.221 & & 0.312 & \\
\hline 5 & 0.159 & 0.6 & 0.230 & 2.2 & 0.328 & 1.2 \\
& 0.158 & & 0.235 & & 0.324 & \\
\hline 6 & 0.190 & 1.1 & 0.290 & 0.0 & 0.330 & 1.9 \\
& 0.188 & & 0.290 & & 0.324 & \\
\hline
\end{tabular}

In equations (2)-(7) $\omega$ is the angular frequency in $\mathrm{rad} / \mathrm{s}$ and the main spectral parameters are described below

- $\sigma_{0}$ represents the conductivity of the DC current, in $\mathrm{S} / \mathrm{m}$.

- $\delta$ is a non-dimensional parameter defined in the interval $0 \leq \delta<1$, which refers solely to the pore path representative of the locus where polarization is produced and involves only the components of the ohmic conduction.

- $\eta$ represents the relative strength between the ohmic component and the diffusion-produced component of the electrical current inside the electrical double layer. Its unit is $s^{-1 / 2}$, and its value ranges from 1 to about 150 .

- $\tau$ corresponds to the relaxation time of the electrical double layer zone, due to the capacitance effect of the system. It is defined in the interval $10^{-7} \leq \tau \geq 10^{-2}$ s.

- $m$ is the "chargeability" (Seigel 1959), defined as a relation involving the asymptotic values of the resistivity, in the interval $0 \leq m<1$.

An analysis of all the parameters can be useful for the definition of the mineral species disseminated in the rock. Initially we employed the inversion procedure to compute the values of Dias' parameters that best matches the observed phase data from measurements on the sea bottom for four arrays spacing of investigation. Figure 10 displays both the theoretical and the experimental phase data employing the inversion algorithm of (Sampaio et al. 1998) applied to the L2 norm described by Porsani et al. (2000). The square error L2 norm is often associated with a Gaussian error distribution (Porsani et al. 2000). Table II shows that the parameters practically do not change for the four array spacing investigated, and that the maximum error of the L2 norm inversion procedure is less than $18 \%$. Then we fitted the observed apparent conductivity values with the modulus of Dias' conductivity function computed with the obtained parameters. Figure 11 shows the comparison employing disk electrodes with a radius of $0.025 \mathrm{~m}$, placed at the sea bottom for electrode spread $n=3$. The maximum difference between the 


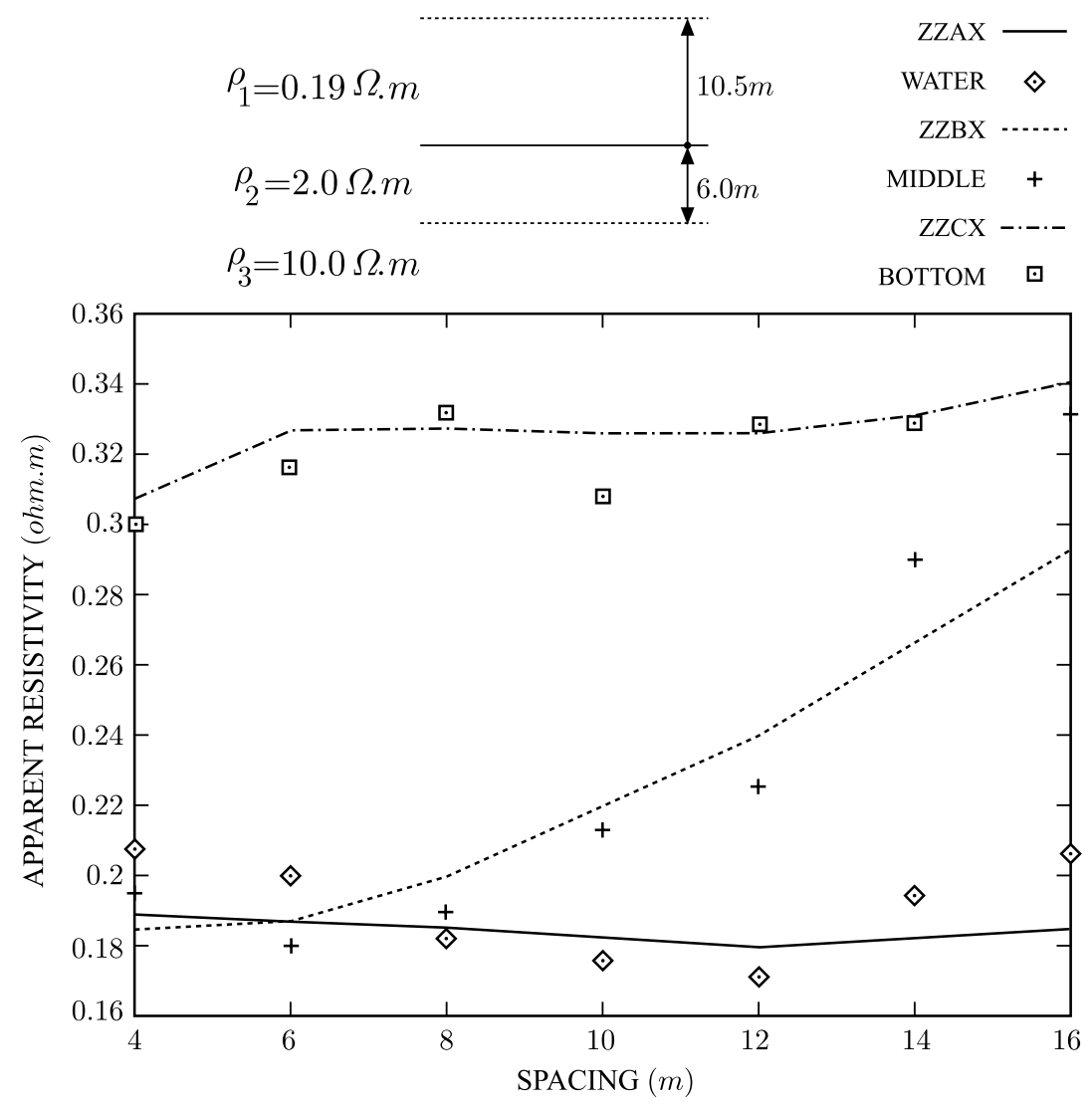

Fig. 8 - Forward modeling of the apparent resistivity for the three sea levels of investigation. ZZAX and WATER represent respectively the theoretical and the experimental data at the sea surface. ZZBX and MIDDLE represent respectively the theoretical and the experimental data at seven meters deep. ZZCX and BOTTOM represent respectively the theoretical and the experimental data at the sea bottom. The experimental data are for $\mathrm{f}=0.25 \mathrm{~Hz}$, and the disk electrode with radius of $0.075 \mathrm{~m}$.

observed and the theoretical data is less than $10 \%$, though the observed conductivity is always larger than the theoretical.

Since each parameter has a physical meaning it is possible to define the dominant polarization phenomenon by making its integrated analysis. Besides a relatively small PFE value, we observe from the data of Table II that: " $\mathrm{m}$ " is relativity large; $\tau$ is close to its minimum value; $\delta$ presents an average value; and $\eta$ presents a relatively large value. A small value of $\tau$ indicates small grain size, and a large value of $\eta$ indicates predominance of the diffusion mechanism. So the electric current flows mainly through the faradaic path, and the phenomenon is predominantly of a membrane polarization type. This is indicative of the presence of argillaceous sands, containing very few if any disseminated metallic minerals.

\section{CONCLUSION}

Electrical sounding proved to be a feasible tool for shallow marine water investigation: both for in situ 


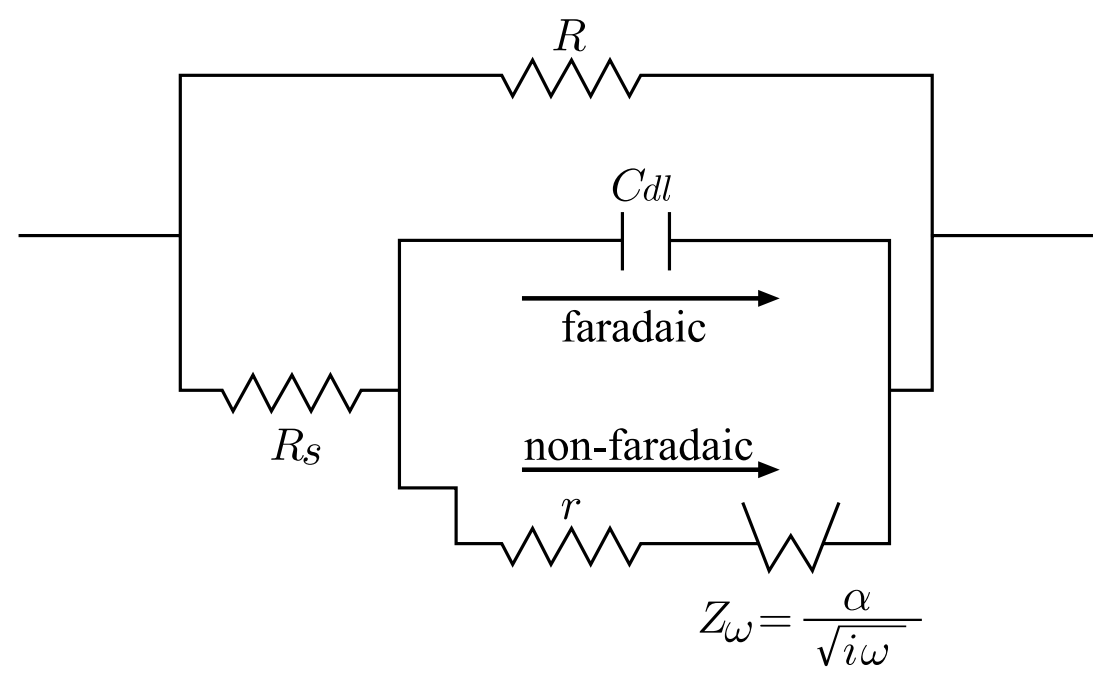

Fig. 9 - Reproduction of Dias' model based on an analog fundamental circuit.

TABLE II

Dias' parameters for the given electrode spreads $n$, employing the disk electrode $(R=0.025 \mathrm{~m})$ at the sea bottom.

\begin{tabular}{c|c|c|c|c|c}
\hline $\mathrm{n}$ & $\mathrm{m}$ & $\delta$ & $\eta$ & $\tau$ & $\%$ of fit \\
\hline 3 & 0.54 & 0.14 & 176.0 & $1.0 \times 10^{-7}$ & 83.6 \\
4 & 0.55 & 0.15 & 177.0 & $1.0 \times 10^{-7}$ & 82.3 \\
5 & 0.55 & 0.13 & 176.0 & $1.0 \times 10^{-7}$ & 82.5 \\
6 & 0.54 & 0.14 & 177.0 & $1.0 \times 10^{-7}$ & 82.7 \\
\hline
\end{tabular}

measurement of the vertical variation of the sea water electrical conductivity; and to define the geoelectric section of the sediments under the sea bottom. The vertical variation is obtained employing the smaller spread $(n=1)$ and positioning the electrodes at different water depths. The geoelectric section is obtained by modeling the complete spread at different water depths. An optimum frequency spectrum between $1.0 \mathrm{~Hz}$ and $32.0 \mathrm{~Hz}$, and a maximum array spacing between the transmitter and the receiver dipoles, $n<6$, were defined. This choice of the measurement parameters restricted both the electromagnetic coupling and the noise caused by movements of the water in an environment with an electrical conductivity of about $5.0 \mathrm{~S} / \mathrm{m}$. The fit applying the L2 Norm to the phase of the apparent resistivity with an error of less than $18 \%$ is not good. But it served for the determination of Dias' parameters, which resulted in a good fit between the theoretical and the experimental curves of the modulus of the apparent conductivity.

The modulus of the apparent resistivity represents an adequate parameter for geoelectric interpretation under the sea. On the other hand the interpretation of spectral induced polarization phenomena employing both the modulus and the phase data defined the type of phenomenon and the source of a low magnitude IP effect in the sediments under the sea bottom. We presented the behavior of only one type of electrode per analysis, because of the relative repeatability of the measurements.

\section{ACKNOWLEDGMENTS}

We acknowledge our fellowships and the grant from $\mathrm{CNPq}$ (Brazilian National Science Foundation). We also thank the support from the divers and the magnetism group of Base Naval de Aratu, Brazil. We are in debt to Prof. I. Mufti for his reading and comment of the manuscript. We also thank Mr. A. dos Santos for providing the computer code for inversion. 
$n=$ array spacing

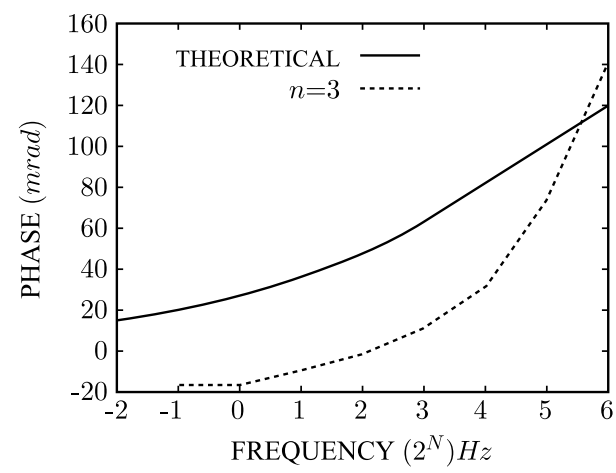

(a)

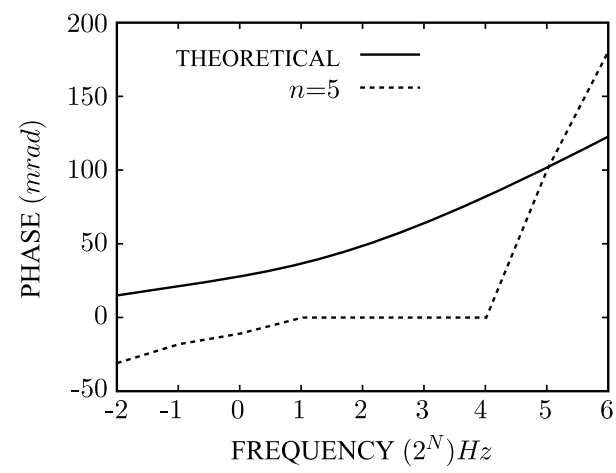

(c)

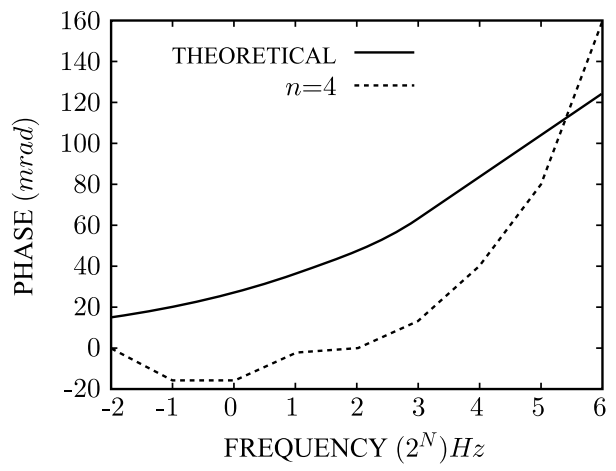

(b)

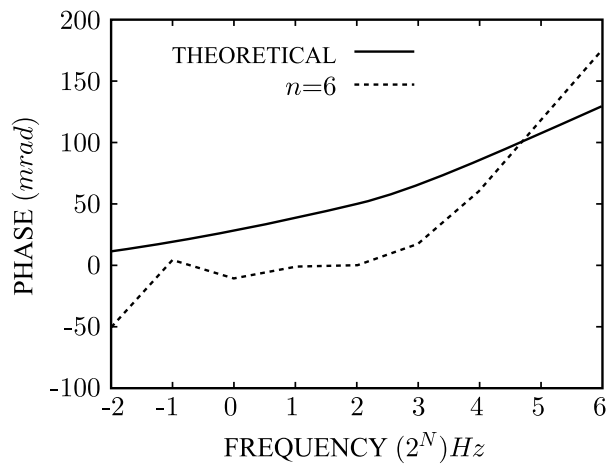

$(d)$

Fig. 10 - Fit between the theoretical and experimental phase data, obtained by inversion of Dias' parameters employing L2 Norm, at the sea bottom: $n=$ array spacing (a) $n=3$; (b) $n=4$; (c) $n=5$; (d) $n=6$.

\section{RESUMO}

Relativamente poucas investigações têm empregado métodos elétricos no ambiente submarino, o qual pode ser promissor para depósitos minerais ou ameaçado por problemas ambientais. Nós medimos o campo elétrico usando eletrodos em forma de disco e de barra na água do mar, em três níveis distintos: superfície, sete metros de profundidade, e fundo do mar a dez metros de profundidade, empregando um dispositivo dipolo-dipolo com $2 \mathrm{~m}$ de afastamento, 7 níveis de investigação e 13 valores de freqüência a intervalos de $\left(2^{\mathrm{N}}\right.$ hertz, $\mathrm{N}=-2,-1,0,1,2$, .. 10). A medida permitiu a análise do campo elétrico como uma função de freqüência e afastamento, e da po- larização induzida espectral. A modelagem e a interpretação da resistividade aparente se ajustaram bem aos dados de perfurações prévias. A análise do espectro da função complexa da resistividade aparente e sua comparação com circuitos equivalentes geraram informações a respeito de tamanho do grão, composição mineral e do aspecto principal do fenômeno de polarização ocorrendo abaixo do fundo do mar. Portanto, o resultado da presente pesquisa mostra ser exequiível medir a variação da resistividade da água do mar, assim como a resistividade dos sedimentos do fundo do mar in situ.

Palavras-chave: resistividade aparente complexa, polarização induzida, modelagem direta, água do mar. 


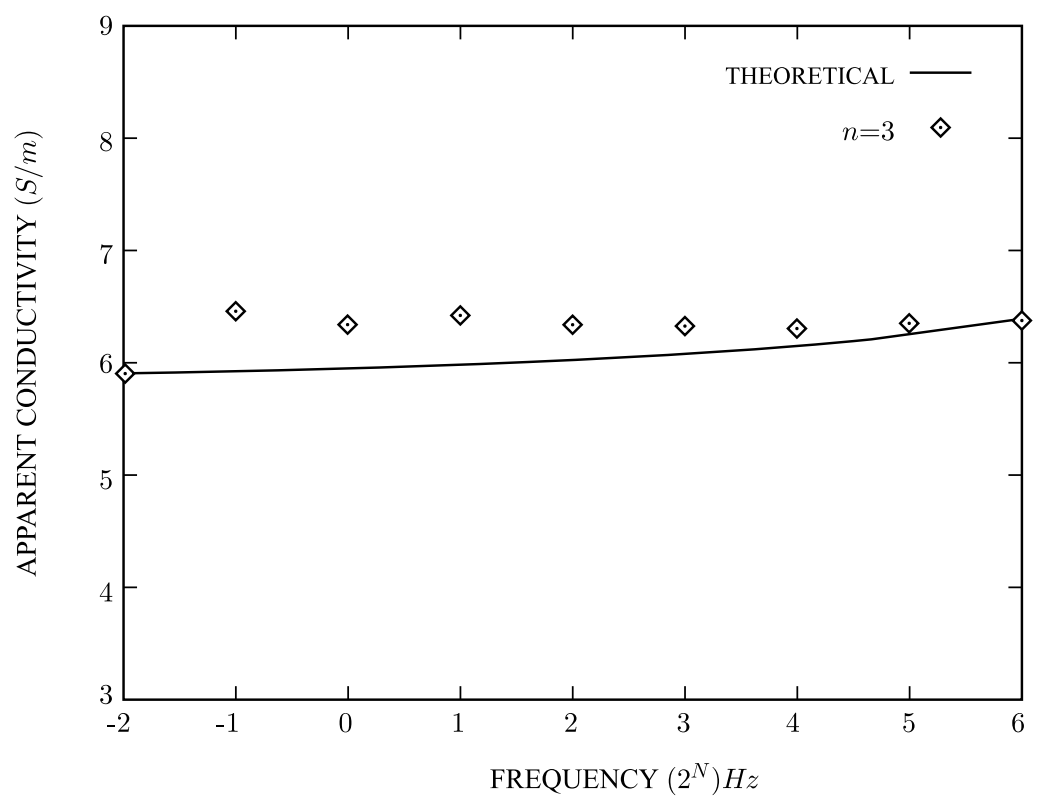

Fig. 11 - Comparison between the experimental and theoretical apparent conductivity $(\mathrm{S} / \mathrm{m})$ with disk electrode $(R=0.025 \mathrm{~m})$ at the sea bottom, for array spacing $n=3$.

\section{REFERENCES}

Baumgartner F. 1996. A new method for geoelectrical investigations underwater. Geoph Prosp 44: 71-98.

Chave AD and Cox CS. 1982. Controlled electromagnetic sources for measuring electrical conductivity beneath the oceans, 1. Forward problem and model study. J Geoph Res 87: 5327-5338.

Chave AD, Constable SC and Edwards RN. 1991. Electrical exploration methods for the seafloor. In: Nabighian MN (Ed.); Electromagnetic methods in applied geophysics, vol II, Applications, Parts A and B. Tulsa: SEG, p. 931-962.

Cheesman SJ, Edwards RN and Chave AD. 1987. On the theory of seafloor conductivity mapping using transient electromagnetic systems. Geophysics 52: 204-217.

Cheesman SJ, Edwards RN and Law LK. 1990. A test of a short-baseline sea floor transient electromagnetic system. Geophys J Int 103: 431-437.
Cheesman SJ, Law LK and Edwards RN. 1991. Porosity determinations of sediments in knight inlet using a transient electromagnetic system. Geo-marine Letters 11: 84-89.

Cole KS And Cole RH. 1941. Dispersion and absorption in dielectrics. J Chem Phys 9: 341-351.

Debye P. 1929. Polar molecules. The Chemical Catalog Co Inc Also (1945) Dover Publications.

DiAs CA. 1972. Analytical model for a polarizable medium at radio and lower frequencies. J Geophys Res 77: 4945-4956.

Dias CA. 2000. Developments in a model to describe low-frequency electrical polarization of rocks. Geophysics 65: 437-451.

Edwards RN AND Chave AD. 1986. A transient electric dipole-dipole method for mapping the conductivity of the sea floor. Geophysics 51: 984-987.

Edwards RN, Law LK ANd DeLaurier JM. 1981. On measuring the electrical conductivity of the oceanic 
crust by a modified magnetometric resistivity method. J Geophys Res 86: 11609-11615.

Hallof PG, Cartwright PA and Pelton WH. 1979. The use of the Phoenix IPV-2 phase IP receiver for discrimination between sulfides and graphite. In: 49Th Annual International Meeting, New Orleans. Expanded Abstract, SEG.

Lima OL and Sharma MM. 1992. A generalized Maxwell-Wagner theory for membrane polarization in shale sands. Geophysics 57: 431-440.

Nabighian MN and Elliot CL. 1976. Negative induced-polarization effects from layered media. Geophysics 41: 1236-1255.

Pelton Wh, Ward SH, Hallof PG, Sill WR AND Nelson PH. 1978. Mineral discrimination and removal of inductive coupling with multi-frequency IP. Geophysics 43: 588-609.

Petiau G and Dupis A. 1980. Noise, temperature coefficient, and long time stability of electrodes for telluric observations. Geoph Prosp 28:792-804.
Porsani MJ, Stoffa PL, Sen M and Chunduru RK. 2000. Fitness functions, genetic algorithms and hybrid optimization in seismic waveform inversion. J. Seismic Exploration 9: 143-164.

Sampaio ES, Santos AB and Sato HK. 1998. Spectral induced polarization and mineral discrimination. In: 68Th Annual International Meeting, New Orleans. Expanded Abstracts, SEG.

Sato HK. 2000. Potential field from a dc current source arbitrarily located in a nonuniform layered medium. Geophysics 65: 1726-1732.

Seigel HO. 1959. Mathematical formulation and type curves for induced polarization. Geophysics 24: 547-565.

WARD SH. 1990. Resistivity and induced polarization methods. In: WARD SH (Ed.); Geotechnical and Environmental Geophysics. Tulsa, SEG.

WYNN JS. 1988. Titanium geophysics - the application of induced polarization to sea-floor mineral exploration. Geophysics 53:386-401. 